\title{
MONITORAMENTO DE FATORES CLIMÁTICOS E SOCIAIS COMO POTENCIAIS AGRAVOS À MORBIDADE DE DENGUE NA CIDADE DE BARRA DO CORDA, MARANHÃO, BRASIL
}

\author{
MONITORING OF CLIMATE AND SOCIAL FACTORS AS POTENTIAL AGAINST \\ DENGUE'S MORBIDITY IN THE CITY OF BARRA DO CORDA, MARANHÃO, \\ BRAZIL
}

Nilson dos Santos Loiola ${ }^{1}$, Alane Oliveira Nascimento ${ }^{1}$, Pauliérica de Sousa Carvalho ${ }^{2}$, Crispim Araújo Neto ${ }^{2}$, Joyce Jhene Rodrigues do Nascimento ${ }^{2}$, Cauana Fabrício Lima Ribeiro ${ }^{1}$

IFMA/ Campus Barra do Corda ${ }^{1}$, UNICENTRO/ BARRA DO CORDA ${ }^{2}$

Abstract

In the last decades, dengue epidemics have been perpetuated as a serious public health problem, especially in countries with tropical climates, such as Brazil, where the climate associated with socio-demographic factors are configured as important factors in the temporal and spatial distribution of dengue transmission. This epidemiological, documentary, descriptive and quali-quantitative research work aimed to analyze dengue among public health problems in the city of Barra do Corda, Maranhão, Brazil, in the period from 2019 to 2020, with the theoretical support of periodicals, books and specific government documents on the topic, and seeking to correlate local socio-environmental and climatic conditions as factors that aggravate the incidence of dengue in the region studied. 718 cases registered in the period by the Notification Archives Information System (SINAN) were analyzed. It was found that the data showed inconsistencies when compared between 2019 and 2020, it is not possible to define an endemic profile, but observing equivalent occurrences between male and female, prevalence in more populated and urbanized neighborhoods (Trezidela and Altamira I) and that the most affected were individuals aged 20 to 34 years. The work demonstrated the possible influence of the COVID-19 pandemic on the actions to prevent the dengue endemic as well as influencing the notification and decision-making work by the responsible public agencies.

Keywords: Dengue. Epidemiology. Risk factors.
Resumo

Nas últimas décadas as epidemias de dengue têm se perpetuado como um grave problema de saúde pública, em especial nos países de climas tropicais, como o Brasil onde o clima associado a fatores sociodemográficos se configuram como importantes fatores na distribuição temporal $e$ espacial de transmissão da dengue. Este trabalho de pesquisa epidemiológica, de caráter documental, descritivo e quali-quantitativo objetivou analisar a dengue dentre os problemas de saúde pública da cidade de Barra do Corda, Maranhão, Brasil, no período de 2019 a 2020, tendo como apoio teórico periódicos, livros $e$ documentos governamentais específicos sobre o tema, e buscando correlacionar condições socioambientais e climáticas locais como fatores agravantes da incidência da dengue na região estudada. Foram analisados 718 casos registrados no período pelo Sistema de Informação de Arquivos de Notificação (SINAN). Verificou-se que os dados apresentaram inconsistências quando comparados entre 2019 e 2020, não sendo possível definir um perfil endêmico, mas observando-se ocorrências equivalentes entre o sexo masculino e feminino, prevalência em bairros mais populosos $e$ urbanizados (Trezidela e Altamira I) e que os mais acometidos foram indivíduos na faixa etária de 20 a 34 anos. O trabalhou demonstrou possivel influência da pandemia do COVID-19 sobre as ações de prevenção à endemia da dengue bem como influenciando nos trabalhos de notificação e tomada de decisões por parte dos órgão públicos responsáveis.

Palavras-chave: Dengue. Epidemiologia.

Fatores de risco. 
A dengue é uma arbovirose (doença viral transmitida por artrópode hematófago) provocada por um vírus de genoma RNA, do gênero Flavivirus e família Flaviviridae, sendo conhecidos quatro sorotipos do vírus dengue (DENV): DENV-1, DENV-2, DENV-3 e DENV-4, que tem como principal sintoma uma evolução febril aguda podendo desencadear quadros hemorrágicos (FORATTINI, 2002). ${ }^{1}$ Esses vírus são transmitidos pela picada de mosquitos infectados, do gênero Aedes (Stegomya), onde a espécie Aedes aegypti é considerada o principal vetor de importância epidemiológica na transmissão da doença (OMS, 2009). ${ }^{2}$

Nas américas o $A$. aegypti encontrou clima e condições favoráveis a uma rápida proliferação, em especial nos grandes centros urbanos, graças a determinantes socioeconômicos como crescimento desordenado e precariedade no abastecimento de água e limpeza urbana, somados a utilização de recipientes para armazenamento de água (que acabam servindo de criadouros para o mosquito nos domicílios), o fluxo populacional e altos índices pluviométricos (BARRETO; TEIXEIRA, 2008; BRASIL, 2002; MACIEL; SIQUEIRA JÚNIOR; MARTELLI, 2008). ${ }^{3,4,5}$

A Estratégia Global para Prevenção e Controle da Dengue, 2012 - 2020, da OMS $(2012)^{6}$ estimou que, a cada ano, ocorreriam cerca de 50 a 100 milhões de novos casos de infecções de dengue e que, aproximadamente, metade da população mundial viveria em áreas onde a dengue já é endêmica, tornando-a uma preocupação global.

Apesar dos constantes esforços da Organização Mundial da Saúde (OMS) e de diversas linhas de pesquisa já desenvolvidas em inúmeros países, a elaboração de uma vacina com potencial imunizante frente aos quatro sorotipos da dengue ainda não foi possível para uma efetiva proteção da população. Tal fato explicita a importância e necessidade de constantes ações profiláticas e intervencionistas por parte do poder público (em especial das equipes que trabalham na linha de frente contra as endemias) para a diminuição da incidência da dengue (SANTOS et al. 2011). ${ }^{7}$

Mendonça $(2009)^{8}$ reforça que, mesmo que a manifestação da dengue, em sua grande maioria dos casos, apresente fatores desencadeadores como as variáveis meteorológicas, que numa soma de processos favorece a proliferação do mosquito transmissor, há que se pensar sobre o ressurgimento da condição infecciosa que tem forte ligação com falhas nos serviços do sistema público de saúde, quesito fundamental na prevenção e controle epidêmico da doença.

A soma dos fatores climáticos e sociais com as falhas no sistema público de saúde tornaram, atualmente, a dengue no mais importante agravo dentre as arboviroses que afetam o homem, por isso é considerada um grave problema de saúde pública, em especial nos países de clima tropical como é o caso do Brasil (FREITAS; CELESTE; MARIA, 2011). ${ }^{9}$

A presente pesquisa teve como foco principal avaliar os determinantes epidemiológicos e ambientais envolvidos na incidência de dengue no município de Barra do Corda - MA por meio de um levantamento de casos notificados da doença no período de janeiro de 2019 a dezembro de 2020.

Foram considerados como objetivos específicos: associar os casos notificados por mês nos anos de 2019 e 2020 com os índices de pluviosidade, temperatura e umidade; caracterizar a distribuição dos casos de forma temporal e espacial dentre os meses do período de referência e os bairros do município de Barra do Corda - MA e correlacionar o número de casos notificados com a faixa etária, gênero e a ocorrência da atual pandemia do COVID - 19.

\section{Metodologia}

Delineamento do estudo:

Tratou-se de um estudo epidemiológico documental descritivo tendo como objeto os casos de dengue provenientes do município de Barra do Corda - MA, notificados por bairros, os quais foram obtidos de dados do Sistema Nacional de Agravos de Notificações SINAN. O corte do estudo compreende dois anos, 2019 e 2020.

\section{Local do estudo:}

A coleta e tabulação dos dados foram realizados na cidade de Barra do Corda, Estado do Maranhão, região centro sul do estado, a 450 $\mathrm{Km}$ da capital São Luís, cidade localizada numa área com característica de Cerrado, com clima quente e úmido e banhada por dois rios perenes o Rio Corda e o Rio Mearim, possuindo uma população de 82.830 habitantes no censo de 2010 e com população estimada em 2020 girando em torno de 88.492 habitantes (IBGE, 2021). ${ }^{10}$

O município de Barra do Corda possui um IDH de 0,606 e mesmo possuindo uma 
Política Municipal de Saneamento Básico e Plano Municipal de Saneamento Básico o município possui um Sistema de Abastecimento de Água local, que capta água do Rio Corda (componente das Bacias Hidrográficas do Itapecuru e Mearim), mas que é operado pela Gerência de Negócios do município de Presidente Dutra (BRASIL, 2013; CAEMA, [20--]). ${ }^{11,12}$

Coleta dos dados:

Os dados epidemiológicos foram disponibilizados pela Secretaria de Vigilância em Saúde (SVS)/ Secretaria Municipal de Saúde Barra do Corda/ MA, obtidos a partir das fichas de notificação do Sistema de Informação de Agravos de Notificação (SINAN) sendo usado, por tanto, informações de dados oficiais e de domínio público, sem a identificação dos pacientes.

Os dados de fatores climáticos foram disponibilizados pelo Instituto Nacional de Meteorologia (INMET)/ Ministério da Agricultura, Pecuária e Abastecimento, sendo estes organizados em gráficos e tabelas para melhor comparação da ocorrência de dengue entre os anos de 2019 e 2020.

Análise dos dados:

Os números de casos notificados foram organizados e analisados com base nas variáveis: índices demográficos (gênero, faixa etária, bairro) e análise temporal (mês e ano de notificação). Tais dados foram correlacionados com dados climáticos como: índice pluviométrico, temperatura e umidade relativa, sendo registro de dados mensais para o período de corte de 2019 a 2020.

Análise estatística:

Foi realizada análise estatística descritiva de distribuição normal dos dados referente aos dois anos pesquisados, com o auxílio dos programas Excel e BioEstat 5.3.

\section{Resultados e Discussão}

\section{Análise por faixa etária}

Durante o período analisado foram notificados 718 casos de dengue com predominância da faixa etária entre 20 e 34 anos (Tabela 01) em ambos os anos estudados (2019 e 2020), onde se destacaram os bairros da
Trezidela e da Altamira I que também apresentaram a faixa etária menor de 01 ano e a partir de 80 anos como as com menos acometimento.

Tabela 1 - Número de casos de dengue por faixa etária. Barra do Corda - MA, 2019 e 2020.

\begin{tabular}{lcc}
\hline \multicolumn{1}{r}{ Faixa Etária } & $\mathbf{2 0 1 9}$ & $\mathbf{2 0 2 0}$ \\
\hline$<1$ & 7 & 4 \\
\hline $1-4$ & 27 & 8 \\
\hline $5-9$ & 54 & 8 \\
\hline $10-14$ & 52 & 16 \\
\hline $15-19$ & 89 & 42 \\
\hline $20-34$ & 201 & 92 \\
\hline $35-49$ & 104 & 73 \\
\hline $50-64$ & 47 & 30 \\
\hline $65-79$ & 26 & 11 \\
\hline 80 e + & 1 & 4 \\
\hline TOTAL & $\mathbf{4 3 0}$ & $\mathbf{2 8 8}$ \\
\hline
\end{tabular}

Fontes: Sistema de Informação de Agravos de Notificação (SINAN), Secretaria de Vigilância em Saúde (SVS), Ministério da Saúde (MS)

Num estudo sobre perfil epidemiológico sobre um município do sudoeste de Goiás, Tannous $(2018)^{13}$ encontrou que a maior incidência entre os anos de 2014, 2015 e 2016, na referida região ocorreu entre 20 a 39 e a menor incidência entre os menores de um ano e acima de 80 anos. Um outro estudo sobre perfil epidemiológico realizado na cidade de São Luís MA, por Fernandes et al. $(2013)^{14}$, a maior frequência de notificações para casos de dengue ocorreu na faixa etária entre 20 e 34 anos, exatamente como o presente trabalho, e dados tabulados por Santos et al. (2009) ${ }^{15}$ mostraram que o maior número de casos de dengue ocorreu na faixa etária entre 20 e 39 anos, os quais se aproximam muito dos resultados desse trabalho.

Diferentemente dos dados anteriormente descritos, Souza e Barata (2012) ${ }^{16}$ ressaltaram que dentre 1.944 casos notificados e analisados na cidade de Cuiabá entre os anos de 2007 e 2008, a incidência alta ocorreu em crianças de 5 a 14 anos e em jovens de 15 a 19 anos.

No município de Belo Horizonte, entre 1 de setembro de 2007 e 31 de agosto de 2010, foram notificados 76.592 casos de dengue, onde nos três anos estudados a mediana de idade variou de 30 a 31 anos e o sexo feminino foi predominante, variando de 54 a 56\% (SILVA et al., 2021). ${ }^{17}$ 
Flauzino et al. (2009) ${ }^{18}$ ao estudarem a heterogeneidade espacial da dengue na cidade de Niterói, RJ, observaram que de 1.212 casos notificados no SINAN entre 1998 e 2006 , as faixas etárias com maior concentração de casos se concentraram no intervalo de $20-29$ anos $(20,5 \%$, 248 casos) e de 30-39 anos (17,7\%, 215 casos).

Neste presente trabalho a população a partir dos 50 anos foi mais acometida nos bairros da Trezidela e Altamira I, ambos em 2019, e no ano de 2020 houve uma maior incidência entre os bairros da Trezidela, Altamira I e Vila Sampaio, sendo que esses dois últimos bairros apresentaram a mesma quantidade de notificações. De modo geral, para todas as faixas etárias, os bairros com uma tendência para maior número de notificações foram a Trezidela e a Altamira I.

Com relação à distribuição da ocorrência de dengue por faixa etária na população do município de São Sebastião, no estado de São Paulo, durante 2001 e 2002, Ribeiro et al. $(2006)^{19}$ verificaram a ocorrência do dengue em todas os intervalos de faixas etárias estudados, com concentração nos intervalos de 20 a 29 anos e de 30 a 39 anos, para ambos os sexos.

Segundo Gonçalves Neto e Rebelo $(2004)^{20}$, todas as idades são susceptíveis ao vírus da dengue, porém estudos demonstram que o padrão mais observado é a ocorrência de casos nas faixas etárias mais elevadas.

Em uma pesquisa abrangente sobre os casos notificados de dengue em treze capitais do país Guimarães e Cunha $(2020)^{21}$, ao analisarem 972 mil casos notificados no período de 2008 a 2017, constataram a predominância de ocorrência entre pessoas do sexo feminino $(57,6 \%)$ e de idade na faixa etária de $20-40$ anos $(57,7 \%)$.

Evangelista, Oliveira e Gonçalves $(2012)^{22}$, ao analisarem os dados epidemiológicos do dengue específicos para o município de Teresina, capital do Piauí e com fluxo constante de pessoas com a cidade de Barra do Corda (cidade alvo do presente trabalho), encontraram 10.142 casos notificados de dengue clássica entre janeiro de 2003 e dezembro de 2007, com predomínio da doença na faixa etária de 20 - 39 anos, além de 133 casos notificados para a forma hemorrágica da doença no período estudado com a prevalência de casos entre a faixa etária de $5-9$ anos de idade.

Mesmo com tais dados apontando para a necessidade de um olhar mais criterioso para ocorrência de dengue entre adultos com faixa etária economicamente ativa, Santos et al. $(2009)^{15}$ relataram em seu trabalho não acreditar que haveria um comportamento padronizado da ocorrência de dengue em função da idade do indivíduo acometido.

\section{Análise por bairro}

Os bairros de maior extensão territorial e mais populosos tiveram maior incidência, sendo eles a Altamira I e Trezidela (Tabela 02), que sinalizaram como sendo os bairros mais problemáticos e que necessitam de ações mais intensivas da vigilância epidemiológica.

Tabela 2 - Número de casos de dengue por sexo em cada bairro. Barra do Corda - MA, 2019 e 2020.

\begin{tabular}{|c|c|c|c|c|c|c|}
\hline \multirow{2}{*}{ BAIRRO } & \multicolumn{3}{|c|}{2019} & \multicolumn{3}{|c|}{2020} \\
\hline & M & $\mathbf{F}$ & Total & M & $\mathbf{F}$ & Total \\
\hline TREZIDELA & 85 & 67 & 152 & 37 & 46 & 83 \\
\hline ALTO DA TREZIDELA & 2 & 4 & 6 & 4 & 3 & 7 \\
\hline CERÂMICA & 15 & 15 & 30 & 11 & 13 & 24 \\
\hline BELO HORIZONTE & 9 & 3 & 12 & 1 & 5 & 6 \\
\hline TAMARINDO & 11 & 7 & 18 & $* *$ & $* *$ & $* *$ \\
\hline VILA MARIANO I & 6 & 9 & 15 & 9 & 5 & 14 \\
\hline VILA SAMPAIO & 22 & 22 & 44 & 18 & 29 & 47 \\
\hline ARATICUM & 8 & 8 & 16 & 8 & 6 & 14 \\
\hline ALTAMIRA I & 57 & 47 & 104 & 33 & 37 & 70 \\
\hline ALTAMIRA ॥ & 9 & 4 & 13 & 2 & 3 & 5 \\
\hline $\mathrm{COHAB}$ & 7 & 6 & 13 & 4 & 2 & 6 \\
\hline MORADAS DO RIO CORDA & 1 & 4 & 5 & 2 & 1 & 3 \\
\hline RESIDENC. MARANATA & 1 & 1 & 2 & 2 & 1 & 3 \\
\hline VILA ALVORADA & $* *$ & $* *$ & ** & 4 & 1 & 5 \\
\hline VILA MARIANO ॥ & $* *$ & $* *$ & ** & 0 & 1 & 1 \\
\hline TOTAIS & 233 & 197 & 430 & 135 & 153 & 288 \\
\hline
\end{tabular}

Fonte: Sistema de Informação de Agravos de Notificação (SINAN), Secretaria de Vigilância

Os bairros da Altamira I e Trezidela, em Barra do Corda - MA, são bairros grandes, em expansão, com intensa atividade comercial e os mais populosos da cidade, o que acarreta maior geração de lixo, utilização de mais reservatórios de água por moradia, uma vez que a cidade sofre com constantes instabilidades no fornecimento de água por parte da estação de captação e distribuição, e tais fatores são propícios para o estabelecimento de criadouros do A. aegypti. 
A Trezidela também é um bairro que vem sofrendo com constantes problemas de alagamentos, principalmente pelo acúmulo de lixo não orgânico, o que dificulta o escoamento das águas pluviais. A intensa geração de lixo está diretamente relacionada a concentração populacional, ineficiência do serviço municipal de coleta e destinação dos resíduos da comunidade, acarretando entupimento de bueiras, acúmulo de água em diversos tipos de reservatórios pelo lixo, podendo resultar na proliferação do mosquito e, consequentemente, nos casos notificados.

É importante considerar também que os bairros Trezidela e Altamira I concentram empresas, escolas, postos de atendimento de serviços públicos diversos, uma diversidade de prestadores de serviços alimentícios, dentre outros, com uma distância média de $5 \mathrm{Km}$ entre ambos, o que impulsionam uma constante e intensa mobilidade da população entre os dois bairros, contribuindo para a circulação do vírus que, somado à propagação do vetor, perpetuam a epidemia de dengue.

Cunha et al. $(2008)^{23}$ ressaltam que a infraestrutura da região e a maior ou menor oferta de serviços públicos (coleta de lixo, manutenção de áreas públicas, subterrâneas e superficiais) podem ser atribuídas às maiores prevalências de infecção por dengue.

O estudo realizado por Sharma et al. $(2014)^{24}$ sobre a difusão do dengue e seu comportamento espacial e temporal na ilha de Trinidad, no Caribe, contribui para os achados na presente pesquisa, onde ao analisarem o comportamento de casos hemorrágicos da doença concluíram que a diminuição da distância espacial média entre os casos se correlaciona com a ocorrência de surto e a proximidade temporal entre os casos sugere a ocorrência de uma epidemia.

Segundo Ribeiro et al. (2006) ${ }^{19}$, bairros centrais são focos da ocorrência de dengue. Em seu estudo no município de São Sebastião, no estado de São Paulo durante os anos de 2001 e 2002, dos 1.691 casos positivos de dengue analisados, $65 \%$ desses ocorreram nos três bairros centrais do município frente a 41 localidades acompanhadas durante a pesquisa.

Corroborando com tal análise, Câmara et al. $(2007)^{25}$ afirmam que o controle da incidência de dengue tornou-se uma tarefa complicada em decorrência da crescente e desordenada urbanização das cidades, a dificuldade de limpeza dos centros urbanos e da ineficiência de destinação correta dos resíduos gerados pela atividade humana, além dos concomitantes problemas nas tentativas de combate ao mosquito $A$. aegypti e a própria falta de conscientização da população.

Como afirmam Ribeiro et al. $(2020)^{26}$ o efeito urbanístico das cidades, apesar de denotar uma expansão da população, também carrega consigo consequências dessa aglomeração de pessoas num mesmo espaço geográfico, todas tentando suprir suas necessidades básicas, até mesmo quando o poder público não as supre, e um dos pontos cruciais nessa corrida pelo "desenvolvimento" é o fornecimento e manutenção do abastecimento de água potável, o que na ineficiência do poder público a população busca compensação com armazenamento prolongado e, muitas vezes, de forma inadequada.

Segundo Foranttini e Brito $(2003)^{27}$ caixas d'água, potes, latas, garrafas, tambores, dentre outras formas de reservatórios de água são muito favoráveis a postura de ovos por $A$. aegypti em fase reprodutiva o que pode ser uma explicação para o alto número de notificações de dengue nos maiores bairros da cidade, podendo ainda ter relação com sua densidade demográfica e o constante armazenamento de água para suprir as necessidades básicas diárias uma vez que a cidade historicamente sofre com a insuficiência de uma precária unidade de captação de distribuição de água.

\section{Análise por sexo}

O presente trabalho apontou que, do total de 430 casos notificados em 2019, 233 $(54,19 \%)$ foram do sexo masculino, e dos 288 casos notificados em 2020, 153 (53,12\%) foram do sexo feminino (Tabela 02), ou seja, para o período de corte do estudo (2019 - 2020) não houve uma confirmação de um padrão para acometimento de um dos sexos uma vez que para cada ano estudado houve a prevalência de um dos sexos e com uma diferença muito pequena com relação ao outro critério analisado, o que não estabelece uma relação entre a notificação de casos e o sexo em nenhum dos anos analisados.

Quanto ao sexo dos casos notificados, estudos realizados nas cidades de Araguaína - TO (Vasconcelos et al. 1993) ${ }^{28}$, Belém - PA (Nascimento et al. 2003) ${ }^{29}$ e Teresina - PI (Monteiro et al. 2009) ${ }^{30}$, estados vizinhos ao estado do presente trabalho (Maranhão), apontaram para uma prevalência de casos entre pessoas do sexo feminino, sendo justificado tal ocorrência em função da permanência da mulher na residência ou peridomicílio, favorecendo o 
contato com criadouros do vetor e gerando maior probabilidade de transmissão de dengue.

Evangelista, Oliveira e Gonçalves $(2012)^{22}$, ao analisarem os dados epidemiológicos do dengue no município de Teresina - PI, encontraram 10.142 casos notificados de dengue clássica e 133 casos de dengue hemorrágica no período entre janeiro de 2003 e dezembro de 2007 e em tal estudo, ambas as formas de dengue acometeram predominantemente o sexo feminino (5.800 - forma clássica e 89 - forma hemorrágica).

Quanto a ocorrência de dengue em relação ao sexo Flauzino et al. (2009) ${ }^{18}$ observaram que do total de 1.212 casos notificados na cidade de Niterói - RJ, entre os anos 1998 e 2006, 57\% eram do sexo feminino (692 casos).

Souza e Barata (2012) ${ }^{16}$ ao estudarem 1.944 casos notificados na cidade de Cuiabá nos anos de 2007 e 2008 relataram que 51\% dos mesmos eram pessoas do sexo feminino, ou seja, um empate técnico que não evidenciou uma correlação estatística significativa para um dos sexos.

RIBEIRO et al. $(2006)^{19}$ demonstraram maior predominância do dengue em mulheres, correspondendo a, aproximadamente, 60\% ( $\mathrm{n}=$ 969) dos 1.691 casos positivos estudados no município de São Sebastião/ SP, no biênio 2001 2002.

Apesar da dengue acometer indivíduos de qualquer faixa etária e de ambos os sexos Gubler $(2001)^{31}$ afirma que existem estudos apontando para uma maior incidência dessa infecção entre mulheres e com uma maior suscetibilidade nas faixas etárias mais elevadas.

A ocorrência de maior número de casos de dengue apontando para um padrão entre pessoas do sexo feminino é relatada por Torres $(2005)^{32}$ ao considerar que tal grupo é mais vulnerável à doença em decorrência das características domiciliares do vetor.

No entanto, com relação ao sexo é importante ressaltar também que a maior concentração de casos entre as mulheres pode ser decorrente de maior uso de serviços de saúde pelas mesmas, resultando em possível viés de notificação (VASCONCELOS et al., 1993). ${ }^{28}$

No entanto, como afirmam Xavier et al. $(2017)^{33}$ no seu estudo sobre a difusão espaço-tempo do dengue no município do Rio de Janeiro, Brasil, no período de 2000-2013, embora exista diversos trabalhos relatando a ocorrência do dengue em função de variáveis explicativas, as epidemias da doença se manifestam de forma diversas, tanto na sua distribuição temporal como geográfica, e variáveis explicativas como as condições de vida, renda, saneamento, entre outras, ajudam a entender a manifestação da doença, entretanto, o dinamismo da difusão do dengue não parece ser diretamente correlacionado a essas variáveis explicativas.

\section{Análise frente aos fatores climáticos}

Pelo fato do Brasil ser um país tropical Braga e Valle (2007)34 ressaltam que os maiores índices de distribuição da dengue ocorrem entre os cinco primeiros meses do ano como uma decorrência do aumento da temperatura e das incidências das chuvas, fatores que influenciam na proliferação do vetor.

Barra do Corda encontra-se na mesorregião Central do Maranhão, apresentando certa constância na temperatura, que varia de $21^{\circ} \mathrm{C}$ e $31,7^{\circ} \mathrm{C}$. De acordo com a classificação de Köppen, o clima é tropical subúmido seco (Aw') com dois períodos bem definidos: um chuvoso que vai de novembro a abril e outro seco, correspondente aos meses de maio a outubro (CORREIA FILHO; GOMES, 2011). ${ }^{35}$

Segundo Keating $(2001)^{36}$ os fatores climáticos como temperatura e pluviosidade têm apresentado relação com os casos de dengue e afetam a sobrevivência, reprodução, distribuição e o número de vetores, durante a estação chuvosa (verão), que se caracteriza pela alta temperatura e grandes índices pluviométricos, além de representar o período de maiores registros da doença. Corroborando com o autor Keating, Forattini (2002) ${ }^{1}$ também defende a possibilidade de controle sobre a transmissão da dengue por meio do conhecimento gerado através da análise dos casos de dengue quando comparados às condições climáticas.

Neste estudo, foram consideradas algumas variações climáticas para relacioná-las com a incidência do número de casos de notificações de dengue. Destaca-se que as variações climáticas representam apenas um dos múltiplos fatores que podem estar relacionados à incidência da dengue que é considerada típica de regiões tropicais por encontrar nesses locais condições ideais para a proliferação e abrangência do principal vetor, o mosquito Aedes aegypti.

No que diz respeito aos casos notificados no ano de 2019, em Barra do Corda, o mês de janeiro apresentou o ápice de casos registrados no ano, totalizando 118 casos de dengue (Tabela 03). É importante frisar que, ao analisar esse dado, deva ser considerado a influência de fatores como a temperatura, o 
índice de pluviosidade e a umidade do ar referente ao mês em questão e ao anterior, haja visto o tempo necessário para que o mosquito chegue a fase adulta e contraia o vírus, bem como para que surjam os primeiros sintomas e seja feito o diagnóstico e registro dos casos.

Tabela 3 - Notificações de dengue por mês nos anos de 2019 e 2020

\begin{tabular}{|c|c|c|c|c|c|c|c|c|c|c|c|c|c|}
\hline \multirow{2}{*}{ ANO } & \multicolumn{12}{|c|}{ MÊS } & \multirow{2}{*}{ TOTAL } \\
\hline & Jan & Fev & Mar & Abr & Mai & Jun & Jul & Ago & Set & Out & Nov & Dez & \\
\hline 2019 & 118 & 41 & 27 & 47 & 61 & 31 & 35 & 23 & 11 & 10 & 18 & 8 & 430 \\
\hline 2020 & 108 & 96 & 57 & 10 & 6 & 3 & 1 & 0 & 4 & 3 & 0 & 0 & 288 \\
\hline
\end{tabular}

Fontes: Sistema de Informação de Agravos de Notificação (SINAN), Secretaria de Vigilância em Saúde (SVS), Ministério da Saúde (MS)

No primeiro semestre do ano, o quantitativo de casos é bem maior que o segundo, o que pode estar relacionado ao período chuvoso, com o aumento pluviométrico, e à umidade ar, que são condições favoráveis para a manutenção de criadouros do mosquito, e ainda para o êxito no clico de vida desse vetor.

Observou-se que os meses de janeiro (118 casos), fevereiro (41 casos), abril (47 casos) e maio (61 casos) foram os que ocorreram as grandes incidências de casos de dengue. Durante esses meses, a temperatura manteve-se na faixa de $27^{\circ} \mathrm{C}$, sendo ideal para proliferação do mosquito $A$. aegypti, como afirma Beserra $(2006)^{37}:$ " [...] a temperatura entre $21^{\circ} \mathrm{C}$ e $29^{\circ} \mathrm{C}$ pode ser considerada propícia para 0 desenvolvimento do mosquito. No entanto, a temperatura não é a única variação climática que deve ser observada. Os meses anteriormente citados apresentaram elevadas taxas de precipitação e umidade, e esses fatos em conjunto são grandes aliados para o ciclo de vida do mosquito e sua expansão geográfica (Gráfico 01).

Gráfico 1 - Casos notificados de dengue frente a fatores climáticos

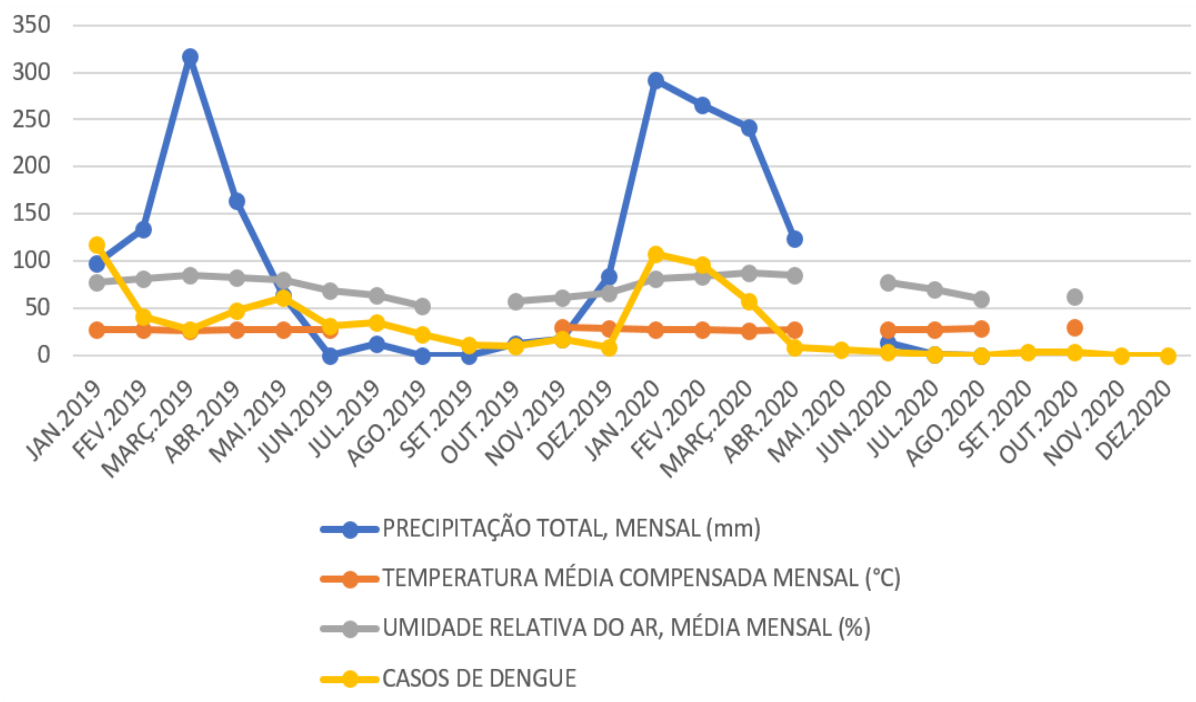

Fonte: Instituto Nacional de Meteorologia (INMET), 2021 
Segundo Silva, Mariano e Scopel $(2008)^{38}$, o A. aegypti encontra condições favoráveis para seu desenvolvimento quando a umidade do ar atinge entre $70 \%$ a $100 \%$, potencializando a ocorrência dos casos. Os meses que apresentaram as maiores taxas de umidade em 2019 foram jan (78\%), fev (81\%), mar (85\%), abril (83\%) e maio (80\%), e coincidem com o período em que ocorreram maiores notificações da doença, exceto o mês de março que, mesmo apresentando, teoricamente, condições propícias para um número maior de casos mostrou uma queda abrupta nas notificações esperadas.

O registro para o mês de março permite inferir, pelo menos, três hipóteses que justificariam os resultados acima, são elas: (1) as variações climáticas aqui destacadas não foram os únicos fatores determinantes para a ocorrência da dengue; (2) pode ter havido subnotificação de casos por parte do sistema público de saúde; (3) os infectados não procuraram tratamento em estabelecimentos oficiais de saúde.

Outra variação a ser considerada são os valores de precipitação, característica do próprio clima da região, que tiveram como registros: jan (98,2 mm), fev $(133,6 \mathrm{~mm}), \operatorname{mar}(317 \mathrm{~mm}), \mathrm{abr}$ $(163,8 \mathrm{~mm})$, mai $(63 \mathrm{~mm})$ e dez $(83,3 \mathrm{~mm})$ e estes meses apresentaram os maiores volumes de chuva e os maiores casos de notificações da doença. Cabe ressaltar que o mês de dezembro de 2019 provavelmente contribuiu para a ocorrência de casos nos meses seguintes, considerando que todas as formas de reservatórios de água parada puderam servir de criadouros para o mosquito proliferar e infectar, após seguir as fases do seu ciclo de vida, desde a postura dos ovos até a infecção de pessoas.

Os estudos de Silva, Mariano e Scopel $(2008)^{38}$ corroboram com o presente trabalho quando destacam que o aumento da chuva permite que reservatórios de água se tornem lugares propícios para os mosquitos depositarem seus ovos, assim sua reprodução não ocorre, exclusivamente, nos meses de grande precipitação, mas sim nos meses seguintes. Outro destaque cabe ao mês de março, que mesmo com temperatura, umidade e precipitação favorável à proliferação e ocorrência da doença não seguiu o parâmetro e apresentou uma redução no número de casos, certamente por fatores não considerados nesta pesquisa, mas que não deixam de ser importantes e não devem ser desprezados.

Nos meses de julho, agosto, setembro e outubro de 2019 não houve registro de dados sobre a temperatura, o que impossibilitou a realização de correlações matemáticas entre notificações e variação de temperatura, no entanto, foi possível observar uma queda no número de casos notificados para os meses citados de 2019. No período de 2019 descrito houve uma queda nos índices pluviométricos e baixa umidade do ar, características do clima tropical subúmido seco (Aw') no período de estiagem, uma possível contribuição para a redução das notificações, assim, à medida que a pluviosidade e a umidade do ar baixam os casos de notificações da dengue também tendem a diminuem. Nos meses de novembro e dezembro de 2019, por exemplo, foram notificados, respectivamente, 18 e 8 casos, números pequenos, considerando os registros do início do mesmo ano.

Para o ano de 2020, além dos fatores climáticos e condições sociodemográficas é necessário considerar a eclosão da pandemia oriunda do COVID - 19, causada pelo novo coronavírus (Sars-Cov-2), visto a rotina atípica que se instalou na sociedade.

Nos três primeiros meses de 2020 os casos notificados corroboram com os registros climáticos e se aproximam da rotina de notificações esperada para o ano com base nos dados de 2019, e apresentou temperatura de $27^{\circ} \mathrm{C}, 27^{\circ} \mathrm{C}$ e $26^{\circ} \mathrm{C}$, precipitações de $291,5 \mathrm{~mm}$, $265,3 \mathrm{~mm}$ e $241,3 \mathrm{~mm}$ com umidade do ar de $82 \%, 84 \%$ e $87 \%$, em janeiro, fevereiro e março, respectivamente, no entanto, de abril até dezembro de 2020 foi observado uma queda abrupta das notificações, com alguns meses sem notificação alguma. A principal hipótese para a redução tão fora do esperado ocorrida, em especial no segundo semestre de 2020 , é de que a rotina de isolamento domiciliar proposta para a sociedade tenha influenciado na falta de notificações ou que tenham se tornado casos subnotificados em virtudes de sintomas análogos ao da COVID-19, por exemplo, somados ao fato de que as recomendações do Ministério da Saúde para as atividades dos Agentes Comunitários de Saúde (ACS), por questões de segurança sanitárias, levaram a suspensão dos trabalhos in loco nos domicílios, retomando as atividades de visitação domiciliar ao final do ano de 2019, mas com visitas bem reduzidas e focadas na área peridomiciliar.

A partir de abril de 2020 observou-se uma queda acentuada no número de casos de dengue registrados pela Secretaria de Saúde além de temperatura de $26^{\circ} \mathrm{C}$, pluviosidade de $123,6 \mathrm{~mm}$ e taxa de umidade do ar $85 \%$, condições que, de acordo com os estudos que já 
existem sobre a doença, são propícias para o avanço da dengue e ideais para o desenvolvimento do ciclo de vida do mosquito, no entanto, o número de casos diagnosticados e registrados ficou muito abaixo dos meses anteriores, totalizando 17 notificações de maio a dezembro de 2020.

Em relação aos meses de maio, setembro, outubro, novembro e dezembro de 2020, por falta de dados referentes aos fatores climáticos dos meses citados, não foi possível correlacionar tais fatores com a incidência de casos da dengue, além disso não foram notificados casos de dengue nos meses de agosto, novembro e dezembro desse mesmo ano.

Em junho, julho e agosto identificou-se uma redução pluviométrica onde, a pequena quantidade de chuva ocorrida é característica do início do período de estiagem, sendo essa redução acompanhada de baixa taxa de umidade do ar e temperatura elevada com destaque para os meses de agosto $\left(28^{\circ} \mathrm{C}\right)$ e outubro $\left(30^{\circ} \mathrm{C}\right)$. Poucos casos da doença foram registrados no segundo semestre de 2020, onde os níveis de temperatura, pluviosidade e umidade do ar apresentaram valores desfavoráveis para proliferação do mosquito $A$. aegypti.

\section{Considerações finais}

Não foi possível associar o aumento ou a redução dos números de casos entre 2019 e 2020 com possíveis fatores agravantes, bem como não foi possível afirmar um perfil epidemiológico da dengue na cidade de Barra do Corda - MA e a obtenção de um real perfil dessa arbovirose. Nesse sentido, várias correlações matemáticas planejadas para o projeto se tornaram inviáveis haja visto, por exemplo, a quantidade de informações sobre os fatores climáticos da cidade de Barra do Corda - MA que não foram registradas em variados meses do ano de 2020.

Com a pesquisa também não foi possível realizar as análises inferenciais previstas para os dados em decorrência de muitas discrepâncias entre as notificações de dengue (2019 e 2020) e a falta de informações, tanto no que se refere às notificações de casos de dengue quanto na coleta de dados climáticos pela estação meteorológica municipal, o que pode ter acontecido ou por uma falta de atividade dos órgão públicos responsáveis por esses dados e/ou por um relaxamento dos cuidados partindo da própria população onde ambos os fatores podem ter ligação direta com a atual pandemia da COVID-19.

A pandemia limitou as atividades laborais, em especial dos Agentes Comunitários de Endemias ( $A C E$ 's), além de que, em virtude do isolamento social, pode ter causado na população um receio em recorrer às unidades de saúde, ou por medo de uma infecção por COVID19 ou por medo de que os sinais e sintomas dos quais se queixaria seriam notificados como uma reação ao coronavírus, indicando infecção diagnosticada por sintomatologia.

Reitera-se que em 2020 a sociedade modificou sua rotina, vivendo um isolamento domiciliar acompanhado de receios que, muitas vezes, possam ter impedido as pessoas de se dirigirem ao atendimento médico, seja para diagnóstico de COVID-19 ou sobre a ocorrência de outras enfermidades, como a dengue, e com a população passando mais tempo no ambiente domiciliar e peridomiciliar e se essas áreas são problemáticas por acumularem diversas possíveis formas de criadouros do mosquito $A$. aegypti, e as mesmas áreas sofreram com a falta e/ou redução das atividades dos ACE's, o esperado para 2020 seria um aumento no número de notificações de dengue, o que não foi confirmado pelos dados obtidos da Secretaria de Vigilância em Saúde do município, que demonstraram a perda de protagonismo da dengue frente a atual pandemia.

Assim, é importante considerar que provavelmente o número de notificações representa apenas uma pequena parte do número de pessoas infectadas. A subnotificação nos registros da doença e o intervalo temporal com ausência de dados sobre as condições climáticas são questões que, também, devem ser considerados.

De uma forma geral também foi possível observar pontos comuns aos demais estudos sobre a dengue no Brasil, como a prevalência em áreas geográficas mais urbanizadas, populosas e com falta de planejamento urbano, como foi evidenciado nos números sobre os bairros Altamira I e Trezidela do presente trabalho. Além de ter sido possível concluir que, no ano de 2019, as notificações demonstraram sofrer influência dos fatores climáticos estudados.

Por fim, é válido também concluir que em Barra do Corda - MA, bem como nos demais municípios brasileiros, a mudança de comportamento da população, principalmente nos bairros mais populosos, é um fator essencial para o controle da dengue uma vez que a participação da população se caracteriza como 
uma ferramenta ativa e eficaz de redução dos focos de criadouros domiciliares do mosquito, devendo ser uma aliada do planejamento e ações das frentes governamentais de controle dessa endemia.

\section{Referências}

1. FORATTINI, O. P. Culicidologia médica: identificação biologia e epidemiologia. São Paulo: Edusp, 2002. 864 p.

2. OMS. World Health Organization. Dengue: guidelines for diagnosis, treatment, prevention, and control: new edition. Genebra: World Health Organization, 2009. 147 p. Disponível em: https://apps.who.int/iris/bitstream/handle/1066 5/44188/9789241547871_eng.pdf?sequence=1\& isAllowed=y. Acesso em: 17 dez. 2020.

3. BARRETO, M. L.; TEIXEIRA, M. G. Dengue no Brasil: situação epidemiológica e contribuições para uma agenda de pesquisa. Estudos Avançados, v. 22, n. 64, p. 53-72, 2008. Disponível em: https://www.scielo.br/scielo.php?script=sci_artt ext\&pid=S0103-40142008000300005. Acesso em: 03 jun. 2020.

4. BRASIL. Ministério da Saúde. Vigilância Epidemiológica. Programa Nacional de Controle da Dengue. Brasília: Ministério da Saúde: 2002. Disponível em: http://bvsms.saude.gov.br/bvs/publicacoes/pncd _2002.pdf. Acesso em: 05 dez. 2020.

5. MACIEL, I. J.; SIQUEIRA JÚNIOR, J. B.; MARTELLI, C. M. T. Epidemiologia e desafios no controle do dengue. Journal of Tropical Pathology, v. 37, n. 2, p. 111-130, 2008. DOI: 10.5216/rpt.v37i2.4998. Disponível em: https://www.revistas.ufg.br/index.php/iptsp/arti cle/view/4998. Acesso em: 12 jan. 2021.

6. OMS. World Health Organization. Global strategy for dengue prevention and control, 2012-2020. Genebra: World Health Organization, $2012 . \quad$ Disponível em: http://apps.who.int/iris/bitstream/handle/10665 /75303/9789241504034_eng.pdf?sequence=1. Acesso em: 27 nov. 2020.

7. SANTOS, F. B.; NOGUEIRA, F. B.; CASTRO, M. G.; NUNES, P. C. G.; FILIPPIS, A. M. B.; FARIA, N. R. C.; SIMÕES, J. B. S.; SAMPAIO, S. A.; SANTOS, C. R.; NOGUEIRA, R. M. R. First report of multiple lineages of dengue viruses type 1 in Rio de Janeiro, Brazil. Virology Journal, v. 387, n. 8, p. 1-4, 2011. Disponível em: https://virologyj.biomedcentral.com/articles/10.
1186/1743-422X-8-387. Acesso em: 09 mai. 2020.

8. MENDONÇA, F. A.; SOUZA, A. V.; DUTRA, D. A. Saúde pública, urbanização e dengue no Brasil. Sociedade \& Natureza, v. 21, n. 3, p. 257269, 2009. Disponível em: https://www.scielo.br/scielo.php?pid=S1982$45132009000300003 \&$ script=sci_abstract $\&$ tlng $=p$ t. Acesso em: 23 ago. 2020.

9. FREITAS, R. M. R.; CELESTE, S. A.; MARIA, C. M. Estratégia intersetorial para o controle da dengue em Belo Horizonte (Minas Gerais), Brasil. Revista Saúde e Sociedade, v. 20, n. 3, p. 773785, 2011. Disponível em: https://www.scielo.br/scielo.php?script=sci_artt ext\&pid=S0104-12902011000300020. Acesso em: 11 jan. 2021.

10. BRASIL. Instituto Brasileiro de Geografia e Estatística - IBGE. População de Barra do Corda - Maranhão. Brasília: Ministério da Economia: $2021 . \quad$ Disponível em: https://cidades.ibge.gov.br/brasil/ma/barra-docorda/panorama. Acesso em: 11 abr. 2021.

11. BRASIL. Ministério das Cidades. Informações sobre Saneamento - SNIS. Brasília, DF, 2013. Disponível em: https://infosanbas.org.br/municipio/barra-docorda-ma/. Acesso em: 16 jul. 2021.

12. Companhia de Saneamento Ambiental do Maranhão/ CAEMA. Gerências de Negócios da Companhia de Saneamento Ambiental do Maranhão. São Luís, [20--]. Disponível em: http://www.caema.ma.gov.br/portalcaema/tem plates/bizdevelopment_blue/mapa/index.html\#p dutra. Acesso em: 16 jul. 2021.

13. TANNOUS, I. P. Perfil epidemiológico e geográfico da infecção pelo vírus da dengue em um município do sudoeste de goiás: um estudo transversal. 2018. 66 f. Dissertação (Mestrado em Ciências Aplicadas à Saúde) - Universidade Federal de Goiás, Jataí, 2018.

14. FERNANDES, D. R. SANTOS, E. A.; ARAÚJO, A. F. D.V.; ZANNONI, C.; SARDINHA, A. H. L.; RODRIGUES, Z. M. R. Epidemiologia da dengue em São Luís - Maranhão, Brasil, 2000 a 2007. Revista Caderno de Pesquisa, v. 20, n. 2, p. 68-75, 2013. DOI: 10.18764/21782229.v20n2p68-77

15. SANTOS, C. H.; SOUSA, F. Y.; LIMA, L. R.; STIVAL, M. M. Perfil epidemiológico da dengue em Anápolis-GO, 2001 - 2007. Revista de Patologia Tropical, v. 38, n. 4, p. 249-259, 2009. DOI: $10.5216 /$ rpt.v38i4.8588

16. SOUZA, L. S.; BARATA, R. C. B. Diferenciais intraurbanos na distribuição de dengue em Cuiabá, 2007 e 2008. Revista Brasileira de Epidemiologia, v. 15, n. 4, p. 761- 

FRUTUOSO, L. C. V.; SAID, R. F. C. Varredura espacial para identificação de áreas de risco epidêmico e fatores associados a dengue: experiência em Belo Horizonte, Minas Gerais. HYGEIA, Revista Brasileira de Geografia Médica e da Saúde, v. 17, p. 14 - 25, 2021. DOI: 10.14393/Hygeia17057163. Disponível em: http://www.seer.ufu.br/index.php/hygeia. Acesso em: 12 jul. 2021.

18. FLAUZINO, R. F.; SOUZA-SANTOS, R.; BARCELLLOS, C.; GRACIE, R.; FIGUEIREDO, M. A.; MAGALHÃES, M.; OLIVEIRA, R. M. Heterogeneidade espacial da dengue em estudos locais, Niterói, RJ. Revista de Saúde Pública, v. 43, n. 6, p. 1035-1043, 2009. DOI: 10.1590/S003489102009005000064. Disponível em: https://www.scielo.br/j/rsp/a/tY77SHbhZzjKw5z X3TXRczL/abstract/?lang=pt. Acesso em: 14 jul. 2021.

19. RIBEIRO, A. F.; MARQUES, G. R. A. M.; VOLTOLINI, J. C.; CONDINO, M. L. F. Associação entre incidência de dengue e variáveis climáticas. Revista de Saúde Pública, v. 40, n. 4, p. 671 - 676, 2006. Disponível em: <http://portal.saude.gov.br/portal/arquivos/kitd engue/epidemiologia/textos/associacaodenguee variaveisclimaticas.pdf >. Acesso em: 15 jul. 2021. 20. GONÇALVES NETO, V. S.; REBÊLO, J. M. M. Aspectos epidemiológicos do dengue no município de São Luís, Maranhão, Brasil, 19972002. Cadernos de Saúde Pública, v. 20, p. 1424 1431, 2004. Disponível em: <http://www.scielo.br/pdf/csp/v20n5/39.pdf>. Acesso em: 18 jul. 2021.

21. GUIMARÃES, L. M.; CUNHA, G. M. Diferenças por sexo e idade no preenchimento da escolaridade em fichas de vigilância em capitais brasileiras com maior incidência de dengue, 2008-2017. Cadernos de Saúde Pública, v. 36 , n. 10 , p. $1-12$, 2020. DOI: 10.1590/0102311X00187219. Disponível em: https://www.scielo.br/j/csp/a/YBDXrLCByWYfxN xk9DnhG8v/?lang=pt. Acesso em: 16 jul. 2021.

22. EVANGELISTA, L. S. M.; OLIVEIRA, F. L. L.; GONÇALVES, L. M. F. Aspectos epidemiológicos do dengue no município de Teresina, Piauí. Boletim Epidemiológico Paulista, v. 9, n. 103, p. 32 - 39, 2012. Disponível em: http://periodicos.ses.sp.bvs.br/scielo.php?script= sci_arttext\&pid=S1806-

$42722012000700004 \& \operatorname{lng}=e n \& n r m=i s o$. Acesso em: 16 jul. 2021. 
da Sociedade Brasileira de Medicina Tropical, v. 36, n. 1, p. 484-485, 2003.

30. MONTEIRO, E. S. C.; COELHO, M. E.; CUNHA, I. S.; CAVALCANTE, M. A. S.; CARVALHO, F. A. A. Aspectos epidemiológicos e vetoriais da dengue na cidade de Teresina, Piauí - Brasil, 2002 a 2006. Revista Epidemiologia e Serviços de Saúde, v. 18, n. 4, p. 365-374, 2009. DOI: 10.5123/S1679-49742009000400006

31. GUBLER, D. J. Human arbovirus infections worldwide. Annals of the New York Academy of Sciences, v. 951, n. 1, p. 13-24, 2001. DOI: 10.1111/j.1749-6632.2001.tb02681.x

32. TORRES, E. M. Dengue. Rio de Janeiro: Editora FIOCRUZ, 2005, 344 p.

33. XAVIER, D. R.; MAGALHÃES, M. A. F. M.; GRACIE, R.; REIS, I. C.; MATOS, V. P.; BARCELLOS, C. Difusão espaço-tempo do dengue no Município do Rio de Janeiro, Brasil, no período de 2000-2013. Cadernos de Saúde Pública, v. 33, n. 2 , p. 1 - 13, 2017. DOI: 10.1590/0102311X00186615. Disponível em: https://www.scielo.br/j/csp/a/YQ5vDJ4QCwf4M C8KGVRVtL/?lang=pt. Acesso em: 13 jul. 2021.

34. BRAGA, I. A.; VALLE, D. Aedes aegypti: histórico do controle no Brasil. Revista Epidemiologia e Serviços de Saúde, v. 16, n. 2, p. 113-118, 2007. DOI: 10.5123/S167949742007000200006

35 CORREIA FILHO, F. L.; GOMES, É. R.; NUNES, O. O.; LOPES FILHO, J. B. Projeto cadastro de fontes de abastecimento por água subterrânea, estado do Maranhão: relatório diagnóstico do Município de Barra do Corda. Teresina: CPRM-Serviço Geológico do Brasil, 2011, 31 p.

36. KEATING, J. An investigation into the cyclical incidence of dengue fever. Social Science \& Medicine Journal, v. 53, n. 12, p. 1587-1597, 2001. Disponível em: https://www.sciencedirect.com/science/article/a bs/pii/S0277953600004433. Acesso em: 03 set. 2020.

37. BESERRA, E. B. Biologia e exigências térmicas de Aedes aegypti (L.) (Diptera: Culicidae) provenientes de quatro regiões bioclimáticas da Paraíba. Neotropical Entomology, v. 35, n. 6, p. 853-860, 2006. DOI: 10.1590/S1519-566X2006000600021

38. SILVA, S. J.; MARIANO, Z. F.; SCOPEL, I. A dengue no Brasil e as políticas de combate ao Aedes Aegypti: da tentativa de erradicação às políticas de controle. Rev. Hygeia, v.3, n.6, p.163175, 2008. Disponível em: http://www.seer.ufu.br/index.php/hygeia/article /view/16906. Acesso em: 07 mar. 2021. 


\section{Endereço para Correspondência}

Nilson dos Santos Loiola

Rua 01, Quadra 03, Casa № 02, Residencial Rio

Corda, Bairro Altamira, Barra do Corda -MA -

Brasil

CEP: $65950-000$

E-mail: nilson.loiola@ifma.edu.br

Recebido em 14/05/2021

Aprovado em 04/08/2021

Publicado em 27/09/2021 